\title{
Las defensas del Reino de Nápoles: el caso de Castellammare di Stabia entre el ' 700 y el ' 800
}

Francesca Castanò

Department of Civil Engineering, Design, Construction and Environment, Second University of Napoles, Italy, francesca.castano@unina2.it

\begin{abstract}
The historical research presents the main projects of interest in redesigning the coast of Castellammare between the 17th and 18th centuries. This port city with the Gulf of Naples and its fortified system view dates the first port expansions between 1715 and 1726. This work continued with the completion of a military arsenal in the Bourbon period, which can be considered an ambitious project during the French decade and subsequent restoration interventions.

He study shows how the redesigning of technical trajectories of the kingdom of Naples behaved under different governments, a willingness to adapt the network of coastal infrastructure and enhancement of the defense systems of military garrisons. Castellammare grew around its port. Industries increased with the creation of the Royal Glassware and commercial activities were developed thanks to the leather factories and the construction of the major cordage. The construction of the first ships and frigates of the kingdom began in the Royal Shipyard developing a defensive system of vanguard. The presence of the port, the old tradition of shipbuilding, the vast beach that stretches behind the quay; are the main reasons for choosing John Acton to establish in Castellammare the Royal Shipyard to manufacture large vessels. In the following years, the urgent necessity to find new spaces for the manufacture of boats, evident urgency at the end of the 17th century, entailed the developing of the first most important project to expand the shipyard during the French decade. From the Murattiano epilogue, the inactivity of the naval army lasts until the twenties of 18th century, when, by order of Fernando I, the works to build the first of the three fixed berths designed by the French people restarted. Successively with Fernando II, the last phase of the works started, being perhaps the most intense modernization of the yard in order to adapt it to the new vapour technologies.
\end{abstract}

Keywords: Reino borbónico, fortificaciones, puerto, astillero

\section{Introducción}

La construcción naval y la actividad portuaria se constituyeron entre los siglos XVIII y XIX como los mecanismos de mayor importancia en el desarrollo de la vida cotidiana y en el impulso urbano de Castellammare di Stabia, uno de los principales centros costeros del golfo de Nápoles (Amirante, Pessolano, 2005; Acampora, 1996; Musto, 2006). A inicios del Setecientos, la ciudad aparece ya dotada de una banchina grande o "muelle grande" que, realizada en época angioina para el amarre de un elevado número de embarcaciones, hacía alarde de una arraigada tradición en la construcción de navíos.

A diferencia de otros centros costeros dedicados por tradición a la actividad ligada al mar y que se desarrollan a lo largo del litoral hasta alcanzar Sorrento; en Castellammare, el progreso de la actividad portuaria y mercantil fue favorecido por un sofisticado sistema fortificado ideado 
para la protección de la ciudad y conectado al sistema defensivo de los territorios limítrofes (Sirago, 2004).

Escala alternativa al puerto de Nápoles, durante la ejecución de los trabajos de reconstrucción dirigidos por Domenico Fontana a finales del Cinquecento, el muelle de "Castello a Mare" fue «accresciuto perché le navi scariche andassero a stare in quel porto nel tempo d'inverno» como describe el propio Fontana (Fontana, 1590).

A comienzos del Seiscientos se inició en Castellammare la construcción de embarcaciones de mayor envergadura para la formación de una armada de navíos de asalto destinados a la protección de la costa del virreino contra los ataques enemigos, a fin de utilizar la flota española en las expediciones oceánicas (Sirago, 1993).

Al mismo tiempo se intensificó el sistema defensivo. El circuito de fortificaciones existentes cubría exclusivamente la línea costera comprendida entre la porta del Quartuccio, construida por los angioinos en el 1364; la vecina porta di Scanzano (D’Angelo, 1990, p. 159) ${ }^{1}$, al Este, y la porta della Fontana Grande, en las cercanías de la torre Alfonsina, para después ascender lo largo de la colina hasta el castillo más antiguo ${ }^{2}$. La nueva estructura fortificada fue levantada por orden de Alfonso de Aragón y realizada por Gentile de Julio y Ruggiero Aurilia (Gambardella, Jacazzi, 2007) alcanzando un gasto total de dos mil ducados. La construcción fue ubicada a la orilla del mar y conectada con el castillo mediante una larga pasarela fortificada (D’Angelo, 1990; Starace, 1992) $)^{3}$.

En el 1635 el virrey Emanuele Fonseca y Zunica hizo levantar en la vecina playa de Pozzano la conocida como Torre di Porticarello a fin de defender el litoral estabiano de las incursiones piratas que eran realizadas desde el frente occidental, favoreciendo así la expansión de la pesca y el desarrollo de la actividad mercantil más allá de la línea del gran muelle que pasó a contar con una posición central en relación al nuevo sistema fortificado.

\section{Los proyectos de la primera mitad del Setecientos}

Los acontecimientos políticos acaecidos a inicios del Setecientos, junto con la creciente importancia del papel de las ciudades, potenciaron un programa de mejora de los sistemas defensivos de la costa napolitana (Russo, 1989) ${ }^{4}$. Se levantaron las fortificaciones de Rovigliano sobre una fortaleza del siglo XVI, así como aquellas de Pozzano, en la zona costera de Sorrento, siguiendo, en ambos casos, los nuevos sistemas defensivos propuestos por Vigliena. Del mismo modo se iniciaron nuevos trabajos de reconstrucción en la zona portuaria que sufría los daños provocados por la continua acumulación de materiales de depósito arrastrados por la red de canales fluviales que llegaban desde las colinas cercanas, provocando, también, graves daños al lecho marino; espacio necesario para el amarre de navíos (Vanacore, 1984). Tras una primera fase de construcción iniciada en el 1715 (D’Angelo, Di Maio, Di Martino 1982; Vanacore, 1984) ${ }^{5}$, en el año 1726 se confió al ingeniero real Giovan Battista Nauclerio el proyecto para las obras de reestructuración del puerto; para su realización, contó con la colaboración de Pietro Vinaccia (Celoro Parancandolo, 1965, p. 49, 84; Vanacore, 1984, pp. 138-140; Castanò, 2008) ${ }^{6}$. En los análisis preliminares se reconocieron, al menos, tres fases diferentes de construcción y ampliación de la estructura: dos antiguas ampliaciones de al menos 230 metros de longitud y 10 metros de ancho relacionadas con la fase seicientesca y una tercera ampliación aún más antigua que, realizada pocos años antes de las ya mencionadas, contaba con 28 metros de longitud y que fue añadida al puerto primitivo, configurándose así una estructura de cerca de 258 metros $^{7}$. El paso siguiente consistió en evaluar la posibilidad real de ampliar el puerto, teniendo en cuenta la dirección de los vientos, la profundidad del lecho marino y sus características; sin obviar, por supuesto, los consejos e impresiones de peritos y marineros expertos (Amirante, 2004). El proyecto ideado por Nauclerio y Vinaccia planteaba la construcción de un nuevo brazo ortogonal de 50 


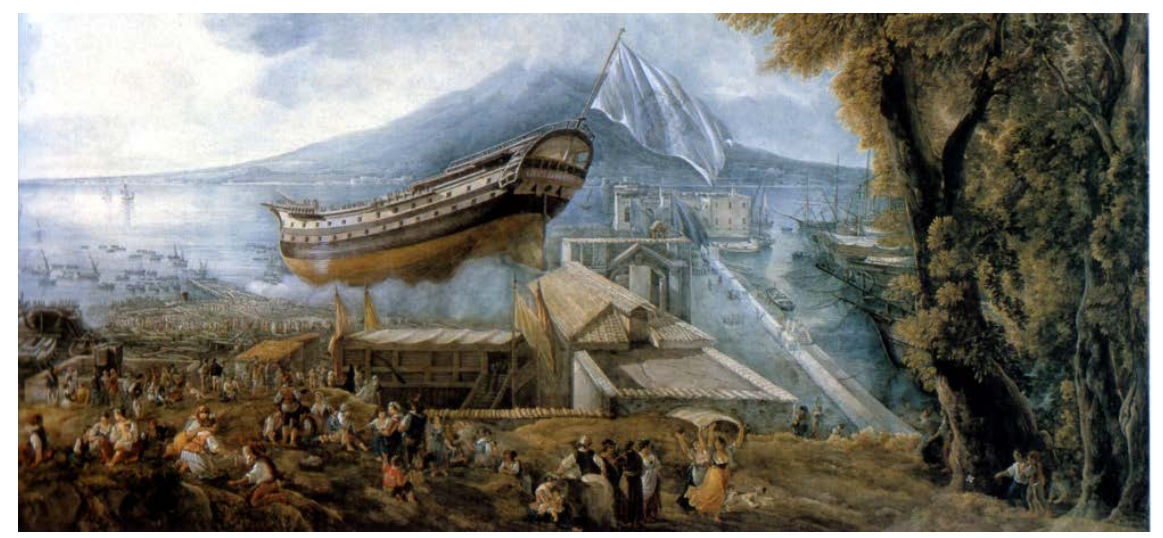

Fig. 1-A. L. R. Ducros, Varo del vascello Archimede, (finales XVIII siglo)

metros que ampliaría el antiguo muelle. Su construcción se realizaría en dos fases diferentes y simultáneas a los trabajos previstos para el lecho marino que preveía un gasto aproximado de 4000 ducados por cada 100 palmos de nueva construcción $^{8}$. Junto con la ampliación de la estructura portuaria se realizaron, como ya había ocurrido en el pasado, nuevas obras destinadas a la mejora del sistema defensivo de la costa, haciendo de Castellammare un punto estratégico en comparación con otros núcleos costeros napolitanos.

La implantación del dominio borbónico comportó un cambio sustancial en las estrategias navales anteriormente planteadas, cambio promovido en buena parte por la influencia de los primeros reformistas. En la totalidad de los puertos del reino, el uso tanto militar como civil de los mismos convivieron de manera natural. La formación de una Segreteria di Guerra e Marina a partir de cuatro departamentos definidos durante el periodo carolino, representó el primer paso hacia esta dirección (Formicola, Romano, 2005). La función de este organismo era la de gestionar las empresas marítimas, los arsenales o la instrucción naval; así mismo, supervisaba a través de "Conferenze di commercio”, temáticas relacionadas con el incremento de intercambios marítimos, el control de la navegación del reino o el control de los principales puertos (Contino, 1983; Zilli, 1990). El aumento de las fuerzas destinadas a la defensa y protección del comercio marítimo representó uno de los puntos principales de la política borbónica. Junto al replanteamiento de las trayectorias náuticas se desarrolló, por lo tanto, la mejora de las infraestructuras costeras a lo largo de las cuales se dispusieron, al mismo tiempo, los nuevos sistemas defensivos. Si inicialmente Carlos se centró en la restauración de los fuertes ya existentes como fuera el caso de Rovigliano, en las proximidades de Nápoles o de Pozzano, en la fachada costera occidental; durante el periodo de regencia sucesivo de Fernando IV, se propuso en Castellammare la costrucción de un sistema defensivo de nueva planta ${ }^{9}$.

Una vez demolida la muralla, la ciudad se expandió más allá de la colina, alcanzando la fachada litoral; aumentaron el número de calles, se potencializó la industria y las playas se poblaron de una actividad mercantil espontánea (Parisi, 1842; Greco 1981).

\section{Las obras reales borbónicas y el nuevo sistema de fortificaciones}

Cuando en el año 1778 John Acton asumió el control de la Segreteria di Marina, Castellammare había ya hecho gala de su producción naviera. No distante de la capital, el rey había recibido ya noticias un año antes de cómo este enclave se configuraba como espacio ideal para establecer «l'Accademia della Guardia Marina, l'officine, l'arsenale, il riposto del legname da costruzione, la fabbrica della tela, la costruzione d'ogni bastimento, il sartiame e li magazzini» ${ }^{10}$. Así, en el año 1783 se iniciaron 
los trabajos para la implantación de los Reales Astilleros. Bajo la dirección del ingeniero Giovanni Bompiede, del ingeniero-constructor Antonio Imbert, del supervisor de los artesanos Andrea Danero, del teniente coronel Salvatore Carraba y del ingeniero Antonio De Simone, encargado de la estimación final, se reconfiguró en un plazo de tres años el perfil de la nueva costa, en la que destacaban los dos brazos paralelos y perpendiculares a la playa y que contaban con la protección del antiguo muelle situado a pocos pasos del recinto que albergaba el área comercial en la zona de la playa en dirección a Pozzano (Rubino, 2005) ${ }^{11}$.

Despues de la construcción de las primeras embarcaciones, el astillero comenzó su máximo nivel de producción a partir de los años noventa, contando con el trabajo de al menos dos mil trabajadores (La Sorsa, 1932; Palumbo, 1972; Radogna, 1979; Formicola, Romano 1990). La mportancia de las nuevas empresas militares impuso el desarrollo de nuevas estrategias defensivas. Entre el 1795 y el 1797 fue colocado en extremo del muelle una batería de artillería fija «capace di 30 bocche di fuoco» y colocadas a dos pisos de altura (D’Ayala, 1847, 309). Única en su género, «la prima nel lido del Mediterraneo», como se lee en la inscripción de la placa conmemorativa (Cosenza, 1901), fue proyectada por el general de artillería francés, Franceso De Pommereul, convocado expresamente por Acton, junto al teniente Giovanbattista Eblè, que dará el nombre a la batería "baja" colocada en la costa en dirección hacia Nápoles.

\section{El decenio francés}

La urgencia por encontrar nuevos espacios para establecer los astilleros, exigencia ya advertida a finales del siglo XVIII, conllevó durante este periodo la creación del primer gran proyecto de ampliación del astillero bajo el dominio muratiano. La línea política de Giuseppe Bonaparte durante los dos años precedentes se había concentrado, sin embargo, en la utilización militar de los puertos existentes. El ingeniero Francesco De Simone estudió la forma de continuar con la ampliación del puerto de Castellammare en el espacio comprendido entre el extremo del viejo muelle y el escollo de Rovigliano, presentando el 7 de julio de 1807 un elaborado estudio al Rey (Tourtier-Bonazzi, 1982; De Divitiis, 1997)12. En la Pianta del Porto e rada di Castellammare realizada a escala de pies parisinos y firmada por el ingeniero hidráulico Giovanni D'Alessio ${ }^{13}$ se pone en evidencia «le quattro visuali», como aparecen ya descritas en el trabajo de De Simone, «procedenti dall'estremità al molo», y en el que aparece señalada la profundidad marina expresada nuevamente en pies parisinos. La misma atención se presta al "forte casamattato" así como a la batería de artillería Eblè y que De Simone consideraba fundamental para la defensa de las embarcaciones y para la seguridad del puerto que, como se puede leer en el estudio presentado al Rey, «resterebbe protetto dalla batteria a doppio ordine costrutta sulla punta del molo e da quelle che dovrebbero costruirsi lungo la costa tra Castellammare e Revigliano, ove ne esiste una comecché nel presente abbandonata» ${ }^{14}$.

Con el ascenso al trono de Gioacchino Murat en el año 1809, los programas políticos fueron modificados. Fueron reorganizadas las estructuras de la zona, con la intención de que pudiesen expandirse sin dificultad alguna hacia la playa que se extendía en dirección a Pozzano, en la zona anteriormente ocupada por el área comercial y que sufría la crisis provocada por el bloqueo continental del tráfico comercial ${ }^{15}$.

El proyecto de ampliación planteado por el propio D’Alessio con la colaboración del ingeniero-constructor Luigi Colbert, modificaba completamente la zona intervenida. Los atracaderos fijos planteados para el amarre de las embarcaciones, de los cuales fueron construidos tres, fueron reconducidos en dirección a la ciudad, llegando a conectarse con el muelle primitivo en aquel lugar donde las aguas eran menos profundas, como había demostrado años antes De Simone. Con esta rotación de 180 grados, los dos técnicos no sólo consiguieron liberar el espacio en torno al antiguo atracadero, 


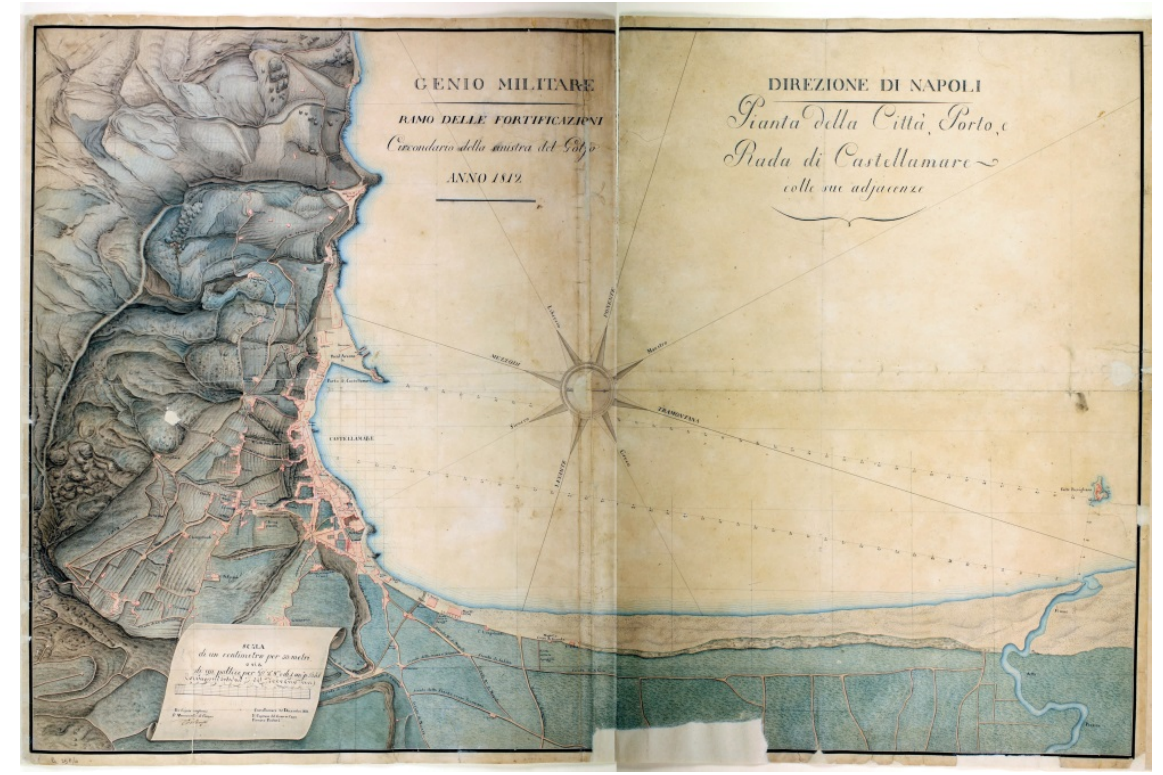

Fig. 2-Genio Militare. Ramo delle Fortificazioni. Circondario della sinistra del Golfo. 1812 (BNN)

si no que con la demolición de aquellas estructuras en desuso, reconfiguraron de manera integral la línea de la costa que vio aumentar su espacio respecto al perfil original. En el Plan du Chantier de Castellamare avec le changemens indiques pour établir trois cales de construction ${ }^{16}$, fue indicado como punto "A" la construcción planteada por D’Alessio y Colbert, y se detallaron los ejes del embarcadero fijo destinado al amarre de las naves. En la misma planta fueron señaladas todas las partes que debían ser conservadas, como las fundiciones, los almacenes adyacentes destinados al depósito del hierro, el forte casamattato o i bagni penali. Igualmente aparecen detalladas aquellas partes cercanas al muelle que debían ser demolidas, así como aquellas zonas destinadas a la edificación ex-novo; en particular las oficinas de servicio y los muros de recinto que definían los nuevos confines del conjunto. En el año 1812 el proyecto ya se había aun iniciado, como evidencia la carta del ministro de Guerra e Marina y que solicitaba la entrega de nuevos espacios para realizar los trabajos ${ }^{17}$. A finales del mismo año tan sólo se había realizado la demolición de una de las partes previstas y ninguno de los atracaderos fijos habían sido realizados como se había planteado en la Pianta della Città, Porto e Rada di Castellammare y en la que aparecen recogidas anotaciones referentes a las estructuras defensivas existentes (Rubino, 2005) $)^{18}$.

\section{Los programas de ampliación en los años de la restauración borbónica}

Tras el epílogo muratiano, la inactividad de la armada naval se mantuvo hasta los años veinte del Ochocientos, cuando por orden de Fernando I se retomaron los trabajos para la realización del primero de los tres embarcaderos fijos programados para el puerto según el proyecto original de D'Alessio y Di Colbert ${ }^{19}$.

Con la subida al trono de Fernando II tiene lugar el inicio de una nueva fase constructiva destinada a modernizar los astilleros para adaptarlos a las nuevas técnicas de construcción de embarcaciones de vapor (Buccaro, Matacena, 2004). La construcción de nuevos almacenes, de un nuevo amarradero, así como de un "bagno penale” de alto rendimiento, conllevó una nueva ampliación que contemplaba la anexión del área comercial y que fue situada en la zona Este, entre el pabellón de la Real Marina y la fábrica de pieles (Rubino, 2005) ${ }^{20}$. 
En el 1780, una comisión de técnicos compuesta por Giovanni Battista Sataiti, Domenico Cuciniello e Giuseppe Mugnai, inició, por orden del rey, las primeras expropiaciones (Formicola, Romano, 1990; Rubino, 2004). Durante los diez años sucesivos supervisaron las obras de reparación del muelle, en el cual se realizaron cuatro nuevos amarraderos de obra, la sistematización de los escollos marinos, obras de restauración, la construcción de nuevos almacenes y la edificación de un faro en la terraza del "forte casamattato", que perdía así cualquier función defensiva para adquirir un nuevo carácter funcional. Mientras tanto, en el año 1842 se instituyeron a Castellammare las escuelas náuticas y entre el 1853 y el 1856 se levanto la nueva cordería destinada a la producción de cordaje para uso náutico, siguiendo los modelos de las fábricas de Marsella, Rochefort y, en particular, de di Tolone ${ }^{21}$.La evolución armoniosa de la actividad constructiva y productiva a mitad del Ochocientos invadía Castellammare desde la fachada litoral que de Pozzano, hasta el nuevo enclave comercial. Mientras tanto, el arsenal que originariamente se había situado a las espaldas de la ciudad, tras el dominio francés pasó a cubrir todo el puerto, convirtiéndolo en una parte integrante del mismo. En las descripciones de algunos viajeros extranjeros, como es el caso de la realizada por Juan Valera bajo el pseudónimo de Silvio Silvis de la Selva, Castellammare parece convertirse en «un jardín y un arsenal», que con «todas sus dependencias y oficinas, causó gran sensación y agradable sorpresa a nuestros marinos», trasladando la atención sobre la locura del puerto, la febril actividad constructiva o las impresionantes embarcaciones (Cirillo Sirri, 1999; Castanò, 2010)22.

Desaparecidas las exigencias defensivas y venido a menos el ideal estratégico de los últimos Borbones, comenzó para Castellammare un periodo de productividad y urbanización en el cual la actividad portuaria y la construcción naval se establecieron como un unicuum bajo la sombra del "castrum maris" que, tras hacerse cargo de la impronta visual de la ciudad, observó impasible el desarrollo de un nuevo enclave obrero e industrial.

\section{Notes}

(1) Archivio di Stato di Napoli (ASN), Pandetta Corrente, F. 1528, fasc. 10235.

(2) Società Napoletana di Storia Patria (SNSP), Fondo Migliaccio, Ms. 8/IV, Cenno cronologico istorico di Quisisana. Dalla sua origine fino ai nostri giorni.

(3) Porta della Fontana Grande, Biblioteca Nazionale di Napoli (BNN), Sezione manoscritti e rari, Ms. XII D 1.

(4) «Avvisi», 1703, p. 137.

(5) Porto e molo di Castellammare. SNSP, Fondo Migliaccio, Ms. 8/VI, fasc. 9. Archivio Storico Comunale di Castellammare (ASC), Fondo Museo, Carte del Notaio D’Aiello, vol. X, f. 224.

(6) ASN, Notai Antichi, Notaio Tommaso Mosca, 28 aprile 1726, F. 275, vol. 1, f. 98 y ASC, Fondo Museo, Carte del notaio d'Aiello, vol. VIII, b. 7, fasc. 5 .

(7) ASN, Notai Antichi, Notaio Tommaso Mosca, 28 aprile 1726, F. 275, vol. 1, f. 98.

(8) Ivi, f. 101 y ff. 99 recto-100.

(9) R. Logerot, Memoria storica scientificopolitico-militare del Regno delle due Sicilie dal 1734 al 1815 del fu cavalier gerosolmitano Don Raffaele Logerot antico uffiziale del corpo Reale d'artiglieria e Genio, cap. V, par. III, SNSP, ms. XXVI.C.6.

(10) ASN, Sezione Militare, Segreteria di Marina, Espedienti, F. 173, f. 37recto.

(11) ASN, Segreteria di Guerra e Marina, anni 1783-1785.

(12) Archivio Nazionale di Parigi (ANP), Archivio di Joseph Bonaparte, 381 AP 10, dossier 2, relación de Francesco De Simone del 7 luglio 1807.

(13) BNN, Sezione manoscritti e rari, Carte geografiche, B.a 29A.66, Giovanni d'Alessio, Pianta del porto e rada di Castellammare di Stabia, 6 luglio 1807. 
(14) ANP, Archivio di Joseph Bonaparte, 381 AP 6, relación de Francesco De Simone del 22 giugno 1807.

(15) Rapporto sullo stato del Regno di Napoli per gli anni 1810-1811 presentato al Re nel suo consiglio di Stato dal ministro dell'Interno Giuseppe Zurlo, in «Monitore delle Due Sicilie», 703, 5 maggio 1813.

(16) BNN, Palat. banc. I.78.11, Plan du Chantier de Castellamare avec le changemens indiques pour établir trois cales de construction.

(17) ASN, Ministero dell'Interno, II inventario, F. 4696, carta de 11 de enero 1812.

(18) BNN, Sezione manoscritti e rari, carte geografiche, B.a 25A.10, 1812.

(19) BNN, Palat. banc. VI.45.4, Pianta del Porto di Castellammare, Cantiere e dipendenze della Real Marina, 1823. BNN, Palat. banc. VI.47.2, Pianta del Porto di Castellammare Cantiere e dipendenze della Real Marina, 1827

(20) BNN, Sezione manoscritti e rari, Carte geografiche, B.a 28.28, Nuovo cantiere mercantile di Castellammare: ivi, B.a 23.10, Pianta del elevato del nuovo Cantiere mercantile compreso tra la fabbrica delle pelli e il forte Eblè.

(21) SNSP, 6.N.3.2, Paolino Fortunato Verzetti, Pianta e prospetto della nuova corderia di Castellammare, 1856.

(22) Esta es una crónica detallada de una excursión de un día a Capri y Castellammare atribuido a Juan Valera, bajo el nombre de Silvio Silvis de la Selva publicado en la revista «El Heraldo» en 1849, cit. in T. Cirillo Sirri, Castellammare "giardino e arsenale" nelle testimonianze letterarie del duque De Rivas e di Juan Valera, in «Annali dell'Istituto Universitario Orientale», Sezione Romanza, XLI, 1, Napoli, Lorientale Editrice, 1999, pp. 15-16.

\section{References}

Acampora A. (1996). Le fonti bibliografiche per la storia di Castellammare di Stabia. N. Longobardi. Castellammare di Stabia.

Amirante G. (2004). "Riflessi sanfeliciani nella produzione matura di Giovan Battista Nauclerio". In Gambardella A. coord. Ferdinando Sanfelice, Napoli e l’Europa. ESI. Napoli, pp. 323-341.

Amirante G., Pessolano M.R. (2005). Immagini di Napoli e del Regno. Le raccolte di Francesco Cassiano de Silva. ESI. Napoli, pp. 107, 146, 239.

Amirante G., Pessolano M.R., coord. (2008). Territorio, fortificazioni, città. Difese del Regno di Napoli e della sua capitale in età borbonica. ESI. Napoli.

Buccaro A. (1992). Opere pubbliche e tipologie urbane nel Mezzogiorno preunitario. Electa Napoli. Napoli, p. 73.

Buccaro A., Matacena G., coord. (2004). Architettura e urbanistica dell'età borbonica. Le opere dello stato, i luoghi dell’industria. Electa Napoli. Napoli, pp. 239-240.

Castanò F. (2008). "Il sistema portuale e le strategie difensive a Castellammare di Stabia tra Settecento e Ottocento”. In Amirante G., Pessolano M.R., coord. Territorio, fortificazioni, città. Difese del Regno di Napoli e della sua capitale in età borbonica. ESI. Napoli, 105-118.

Castanò C. (2010). "Un jardìn y un arsenal. Il porto e il cantiere di Castellammare di Stabia nel Regno di Napoli”. In «Rivista Aipai. Patrimonio Industriale», a. IV, 5, pp. 8-13.

Cirillo Sirri T. (1999). “Castellammare "giardino e arsenale” nelle testimonianze letterarie del duque De Rivas e di Juan Valera”. In «Annali dell’Istituto Universitario Orientale». Sezione Romanza, XLI, 1. L’orientale Editrice. Napoli, pp. 15-16.

Contino E. (1983). Le funzioni dei consoli e lo sviluppo del commercio marittimo del Regno di Napoli. Giannini. Napoli.

Cosenza G. (1901). “Opere d'arte del circondario di Castellammare di Stabia”. In «Napoli Nobilissima», X/IX, pp. 142-143.

D’Angelo G. (1990). I luoghi della memoria. Il centro antico di Castellammare di Stabia. Eidos. Castellammare di Stabia, pp. 62-63.

D’Angelo G, Di Maio M., Di Martino A. coord. (1982). L'archivio storico comunale 1513-1946. Castellammare di Stabia. 
D’Angelo G., Vanacore C. (1983). Il cantiere navale di Castellammare di Stabia 1783-1983: bicentenario della fondazione. Castellammare di Stabia, pp. 5-38.

D’Ayala M. (1847). Napoli militare. Napoli.

De Divitiis M. R. (1997). "L'archivio privato di Joseph Bonaparte nell Archives Nationales de France e altre fonti per lo studio del "decennio francese" nel Regno di Napoli tra il 1806 e il 1808". In Il futuro della memoria. Atti del Convegno internazionale di studi sugli archivi di famiglie e di persone. (Capri 9-13 settembre 1991). Ministero per i beni culturali e ambientali. Roma, pp. 629635.

de Tourtier-Bonazzi C. (1982). Archives de Joseph Bonaparte, roi de Naples puis d'Espagne (381 AP). Archives Nationales. Archives Nationales. Paris.

Fontana D. (1590). Della trasportazione dell'obelisco vaticano e delle fabbriche di N. S. Papa Sisto V. Roma, libro II, c. 26r., ripr. facs. Carugo A. coord. (1979). Il polifilo. Milano 1979.

Formicola A., Romano C. (1990). "Il periodo borbonico (1734-1860)". In Fratta A. coord. La fabbrica delle navi. Storia della cantieristica nel Mezzogiorno d'Italia. Electa Napoli, Napoli, pp. 75-102.

Formicola A., Romano C. (2005). Storia della marina da guerra dei Borbone di Napoli 1734-1767. I. Ufficio Storico della Marina Militare. Roma, pp. 21-25.

Gambardella A., Jacazzi D. coord (2007). Architettura del classicismo tra Quattrocento e Cinquecento. Campania saggi. Gangemi. Roma, pp. 27-28.

Gambardella A., Jacazzi D. coord (2007). Architettura del classicismo tra Quattrocento e Cinquecento. Campania ricerche. Gangemi. Roma, pp. 166-171.

Greco G. (1981). Stabiae dalle origini ai Borboni. A. Fiory Edizioni. Napoli, pp. 276-277.

La Sorsa S. (1932). "Il Real Cantiere di Castellammare di Stabia”. In Le vie d'Italia. TCI. Milano, pp. 275-283.

Musto G. (2006). “La città di 'Castrum maris de Stabia’ nelle vedute del VVIII e XIX secolo”. In de Seta C. e Buccaro A. coord. Iconografia delle città in Campania. Napoli e i centri della provincia. Electa Napoli. Napoli, pp. 303-325.

Palumbo M. (1972). Stabiae e Castellammare di Stabia. Antologia critica. Aldo Fiory Edizioni. Napoli, pp. 343-344.

Parisi C. (1842). Cenno storico-descrittivo della città di Castellammare di Stabia. Firenze, pp. 43-44.

Radogna L. (1979). Storia della marina militare delle Due Sicilie. Mursia. Milano, pp. 29-30.

Rubino G. E. (2004). Le fabbriche del sud. Architettura e archeologia del lavoro. Giannini. Napoli.

Rubino G. E. (2005). "I cantieri navali di Castellammare di Stabia e lo yacht reale di Francesco I (1828)”. In Rubino G. E., coord. Costruttori di opifici / Millwrights. Architetture del lavoro fra tradizione e innovazione. Giannini. Napoli, pp. 23-37.

Russo F. (1989). La difesa costiera nel Regno di Napoli dal XVII al XIX secolo. Stato maggiore dell'esercito Ufficio storico. Roma, p. 204.

Schiappoli I. (1940). La Marina degli Aragonesi di Napoli. Napoli 1940.

Simoncini G. coord. (1993). Sopra i porti di mare. II. Il Regno di Napoli. L. S. Olschki. Firenze.

Sirago M. (1993). "Attività economiche e diritti feudali nei porti, caricatoi ed approdi meridionali tra XVI e XVIII secolo”. In Simoncini G. coord. Sopra i porti di mare, II, Il Regno di Napoli. Leo Olschki Editore. Firenze, pp. 345-346.

Sirago M. (2004). Le città e il mare. Economia, politica portuale, identità culturale dei centri costieri del Mezzogiorno moderno. ESI. Napoli, p. 13.

Starace F. (1992). "Castellammare di Stabia feudo dei Farnese. Una planimetria del litorale con la «Porta della Fontana Grande» in un libro di disegni di architettura della Biblioteca Nazionale di Napoli”. In «Cultura e territorio». a. IX, pp. 81-90.

Vanacore C. (1982). Castellammare di Stabia nel suo porto, dalle origini al secolo XIX. Napoli, pp. 3334.

Vanacore C. (1984). "Il porto di Castellammare nel secolo XVIII”. In «Cultura e territorio», a. I, 1984, pp. 134-140.

Vanacore C. (1987). Il cantiere navale di Castellammare di Stabia 1780-1983. EDI. Napoli, pp. 13-16.

Zilli I. (1990). Carlo di Borbone e la rinascita del Regno di Napoli. Le finanze pubbliche 1734-1742. ESI. Napoli, pp. 169-171. 